\title{
Giant enhanced optical nonlinearity of colloidal nanocrystals with a graded-index host
}

\author{
J. J. Xiao* \\ Department of Physics, The Chinese University of Hong Kong, \\ Shatin, New Territories, Hong Kong, China \\ K. W. $\mathrm{Yu}^{\dagger}$ \\ Department of Physics, The Chinese University of Hong Kong, \\ Shatin, New Territories, Hong Kong, China and \\ Institute of Theoretical Physics, The Chinese University of Hong Kong, \\ Shatin, New Territories, Hong Kong, China
}

(Dated: October 13, 2018)

\begin{abstract}
The effective linear and third-order nonlinear optical properties of metallic colloidal crystal immersed in a graded-index host fluid are investigated theoretically. The local electric fields are extracted self-consistently based on the layer-to-layer interactions, which are readily given by the Lekner summation method. The resultant optical absorption and nonlinearity enhancement show a series of sharp peaks, which merge in a broadened resonant band. The sharp peaks become a continuous band for increasing packing density and number of layers. We believe that the sharp peaks arise from the in-plane dipolar interactions and the surface plasmon resonance, whereas the continuous band is due to the presence of the gradient in the host refractive index. These results have not been observed in homogeneous and randomly-dispersed colloids, and thus would be of great interest in optical nanomaterial engineering.
\end{abstract}

PACS numbers: 78.66.-w, 78.67.Bf, 78.67.Pt

\footnotetext{
* Electronic address: jjxiao@phy.cuhk.edu.hk

$\dagger$ Electronic address: kwyu@ @hy.cuhk.edu.hk
} 
There have been considerable interests in searching for materials with a large nonlinear susceptibility. The most common way to achieve this is to use composite materials in which the constituent components possess large intrinsic nonlinear responses. Noble metal (typically gold, silver and copper) is often chosen as an ingredient due to their extremely large and fast nonlinear optical response. Many different microstructures have been exploited in an attempt to access the intrinsic optical nonlinearity of metals, for example, in random metallodielectric composites, $, 1,2$ fractal films, ${ }^{2}$ and alternative bilayers, ${ }^{\frac{3}{3}}$ etc. They basically rely on the enhanced local fields in space or on the effectively lengthened scale of the interactions between the matter and the incident light field. However, there is also a great demand for particular optical materials in devices applications, which would benefit from additional tunability of the optical properties. Recently, we studied graded composites, which provide an extra degree of freedom for controlling the optical properties of these materials. ${ }^{4}$ In fact, there exist in nature abundant graded materials, such as biological cells ${ }^{\frac{5}{-}}$ and liquid crystal droplets. ${ }^{6}$ Furthermore, many artificially-graded-index optical metamaterials and elements have been fabricated nowadays. ${ }^{7}$

Colloidal crystal has been extensively studied in nanomaterials engineering and its potential applications range from nanophotonics to chemistry and biomedicine. ${ }^{8}$ It is desirable and of interest to use dielectric-coated metallic nanoparticles with varying shell thickness to form a dielectric constant gradient. $\frac{9}{}$ In this Letter, we theoretically investigate a metallic colloidal crystal immersed in a graded-index host and demonstrate a giant enhanced optical nonlinearity band, which is controllable by the gradient and by the easily-tunable colloidal structure as well. These colloidal crystals can be prepared via layer-by-layer self-assembly, templated sedimentation, methods based on capillary forces, and electrokinetics. ${ }^{8,9,10,11,12}$ We basically use the quasistatic point-dipole approximation, which suffices in terms of characterizing both the gradient effects and the lattice effects, otherwise the solution is formidable, either from a Green's function formalism or from first-principles. 13

The theoretical calculations are deployed on a model tetragonal lattice with uniaxial anisotropy $^{14}$ (see Fig. 1). As examples, let us focus on the body-centered-tetragonal (bct), the body-centered-cubic (bcc) and the face-centered-cubic (fcc) lattices respectively. Among the three cases bcc lattice has the lowest packing density while fcc lattice has the highest one. ${ }^{14}$ Extensions to other colloidal structures such as simple tetragonal lattices are straightforward and similar results are expected. Taking advantages of the normalized interlayer interaction tensor $\mathbf{T}$ (i.e., $\mathbf{T}_{i j}$ denotes the interaction strength between two in-plane dipole arrays) given by the Lekner summa- 
tion method, ${ }^{15}$ we solve the following self-consistent equation for the layer dependent local field $\mathbf{E}_{i}$

$$
\mathbf{E}_{i}=\frac{1}{a^{3}} \sum_{j=1}^{N} \mathbf{T}_{i j} \cdot\left(\alpha_{j} \mathbf{E}_{j}\right)+\mathbf{E}_{i}^{(0)},
$$

where $a$ is the lattice constant ( $b$ and $c$ are the other two lattice constants) as shown in Fig. 11, $\alpha_{j}$ is the layer-dependent linear bare polarizability, here $i, j$ label the crystal layer and $N$ denotes the total layer number. Note $\mathbf{E}_{i}^{(0)}$ in the self-consistent equation is not simply the externally applied electric field $\mathbf{E}_{0}$ due to the presence of gradient. However, it is the field inside the graded host medium, which is thus determined by virtue of the continuity of the normal component of the electric displacement $\mathbf{D}$ in the longitudinal case (i.e., $\mathbf{E}_{0}$ parallel to the uniaxial axis). It is the $z$-axis as shown in Fig. 1] in our case. Nevertheless in the transverse case (i.e., $\mathbf{E}_{0}$ perpendicular to the uniaxial axis), we exactly used the applied field $\mathbf{E}_{0}$ because the boundary condition now becomes the continuity of the tangential component of electric field. We shall compare the effective linear and nonlinear optical responses of colloidal crystals with the different lattice structures (e.g., bct, bcc, and fcc), made of metallic nanoparticles of linear dielectric constant $\epsilon_{1}$ and third-order nonlinear susceptibility $\chi_{1}$, suspended in a host fluid of $\epsilon_{m}$ (see Fig. 1). Both the longitudinal (L) and transverse (T) results will be discussed. The self-consistent equations over $i=1,2 \cdots, N$ are then combined together to take into account the lattice effect and are being able to be transformed into a matrix form as $\mathbf{E}=\mathbf{T A E}+\mathbf{E}^{(0)}$. More precisely, in the longitudinal and the transverse cases, $\mathbf{E}=\left\{E_{i}^{(L, T)}\right\}$ is simply N-dimensional vector and $\mathbf{A}$ is $N \times N$ diagonal matrix of the polarizability, which relates the induced dipole moment $\mathbf{p}_{i}$ of the particle and the local field $\mathbf{E}_{i}$ in the layer $\ell_{i}$, and in fact consists of isotropic linear and nonlinear contributions. That is $\mathbf{p}_{i}=\alpha_{i} \mathbf{E}_{i}+\beta_{i}\left|\mathbf{E}_{i}\right|^{2} \mathbf{E}_{i} / 3$, where $\alpha_{i}=\epsilon_{m} r^{3}\left(\epsilon_{1}-\epsilon_{m}\right) /\left(\epsilon_{1}+2 \epsilon_{m}\right)$. Here $r$ is the radii of the metallic nanoparticles. In the case of weak nonlinearity in the colloidal particles, i.e., $\chi_{1}\left|\mathbf{E}_{i}\right|^{2} \ll \epsilon_{1}$ in the nonlinear relationship $\mathbf{D}_{i}=\epsilon_{1} \mathbf{E}_{i}+\chi_{1}\left|\mathbf{E}_{i}\right|^{2} \mathbf{E}_{i}$, by the perturbation expansion method, ${ }^{16}$ one has

$$
\beta_{i}=\left(\frac{3 \epsilon_{m}}{\epsilon_{1}+2 \epsilon_{m}}\right)^{2}\left|\frac{3 \epsilon_{m}}{\epsilon_{1}+2 \epsilon_{m}}\right|^{2} r^{3} \chi_{1} .
$$

It is noteworthy that the linear local fields $\mathbf{E}_{i}$ around the particles in the layer $\ell_{i}$ are actually obtained by assuming no intrinsic nonlinear response, i.e., we set $\chi_{1}=0$ for solving the selfconsistent equations [Eq. (1)], which is appropriate provided that the nonlinear responses are much less than the linear ones. Next we use the resultant linear local fields $\mathbf{E}_{i}$ to extract the enhancement 
factor of the effective nonlinear susceptibility $\bar{\chi}_{1}$

$$
\gamma \equiv \frac{\bar{\chi}_{1}}{\chi_{1}}=p \frac{\left\langle\left|E_{i}\right|^{2} E_{i}^{2} \beta_{i}\right\rangle}{3\left|\mathbf{E}_{0}^{4}\right| \chi_{1}},
$$

where $p$ is the filling fraction of the metallic colloidal nanoparticles. If the nanoparticles are touching, i.e., with the geometric constraint $a^{2}+b^{2}+c^{2}=16 r^{2}$ (see Fig. 1), then $p=\pi\left[\left(q^{3}+2\right) / q\right]^{3 / 2} / 24$ where $q=(c / a)^{2 / 3}$ quantifies the degree of anisotropy of the periodic lattice ${ }^{14}$ and also determines the normalized interlayer interaction $\mathbf{T}$. This results in structure-controllable optical properties. Note that the average $\langle\ldots\rangle$ in Eq. (3) is taken over the layers $\ell_{i}(i=1, \cdots, N)$ instead of over the nanoparticles spatial volume, because in the dipole approximation the local fields inside each of the particles are homogeneous. We also assume no nonlinear response in the host, which is in fact relatively neglectable comparing to that in the metal. Additionally, a gradient of the dielectric constant of the host fluid is introduced along the uniaxial direction of the colloidal crystal, i.e, $\epsilon_{m}=\epsilon_{m}\left(z_{i}\right)$ in our case, where $z_{i}$ represents the $z$-coordinate of layer $\ell_{i}$. In this regard, we treat the host as a continuously-layered film, thus explicitly has $\mathbf{E}^{(0)} \equiv\left\{E_{i}^{(0)}=E_{0} / \epsilon_{m}\left(z_{i}\right)\right\}$ in the longitudinal case. The formation of the gradient in the host might be achieved by dispersing different polymers in it, by selectively filling with microfluidic materials, ${ }^{18}$ or induced by the presence of a temperature gradient, etc. One can also simply coat the nanoparticles with different coverage shells. But it still remains a challenge because the novel properties from our prediction require a reasonably large gradient in the dielectric constant of the host.

Figure 2 shows in logarithmic scale the optical absorption, i.e., the imaginary part of the effective linear dielectric function $\operatorname{Im}\left(\epsilon_{\text {eff }}\right)$ (upper panels) and the modulus of the nonlinearity enhancement factor $\gamma$ (lower panels) defined in Eq. (3), as functions of the reduced frequency. We specifically compare the results of bct $(q=0.87358)$, bcc $(q=1.0)$, and fcc $(q=1.25992)$ as shown in the three columns for $N=25$ layers. The dielectric function of the metallic colloidal nanoparticles is simply adopted as the Drude form $\epsilon_{1}=1-\omega_{p}^{2} /\left(\omega^{2}+i \omega \Gamma\right)$, while the graded dielectric constant in the host medium is assumed as $\epsilon_{m}\left(z_{i}\right)=1.0+1.25 i / N$ for $i=1,2, \cdots N$. The presence of the inhomogeneity in the host fluid obviously leads to a broadened and giant enhanced resonant band (solid lines) in the low-frequency region. This is interesting for potential telecommunication applications. The results of the same colloid crytal suspended in homogeneous host medium with $\epsilon_{m}=1.0$ (dotted lines) or $\epsilon_{m}=2.25$ (dashed lines) are also presented, in an attempt to demonstrate that the broadened resonant band in some sense stems from the hybridization of the non-graded structures. From the absorption spectrum and the enhancement in the third-order 
nonlinear susceptibility, we would expect an attractive figure of merit. ${ }^{4}$ That is, the materials effectively exhibit large nonlinearity and relatively small absorption. This is certainly superior to pure metal because it generally has large nonlinearity and unwanted absorption concomitantly. In fact, the optical absorption arises from the surface plasmon resonance, which is obtained from the imaginary part of the effective linear dielectric constant $\epsilon_{\text {eff }}$ that is extracted from the generalized Clausius-Mossotti formula. ${ }^{13,14}$ Note that the plasmon resonant peaks in the cases of homogeneous host with periodic arrangment of metallic particles, i.e., the peaks in the dashed and dotted curves in Fig. 2] are red-shifted with respect to the corresponding ones (not shown) of a homogeneous host with randomly-dispersed nanoparticles predicted by the Maxwell-Garnett theory. We actually set a gap (e.g., coated layer thickness in experiments) of $2 a / 5$ between the nearest in-plane lattice particles in order to avoid severe complications arising from the multiple image interactions ${ }^{13,19}$ Thus we have the metal filling fraction $p=(3 / 5)^{3} \pi\left[\left(q^{3}+2\right) / q\right]^{3 / 2} / 24$, e.g., $0.15080,0.14692$, and 0.15994 for the bct, bcc, and fcc lattices, respectively. The introduction of this gap indeed makes the nanoparticles size, and thus the dipole factor relatively small and somewhat unfavorably suppresses the effect arising from variation of the lattice structure, as seen in Fig 2

Furthermore, due to the fact that we treat the continuous variations of dielectric function in the host as layered ones in obtaining $\alpha_{i}$ and $\beta_{i}$, and the fact that the dipoles are actually distributed in discrete lattice nodes, a series of sharp peaks are also observable in Fig. 2, The peaks are merged in the broadened band and become more notable for increased gradient in the host dielectric constant (not shown), whereas they tend to disappear as the number of crystal layers $N$ is increased. This is also understandable in the generalized Bergman-Milton spectral representation in graded

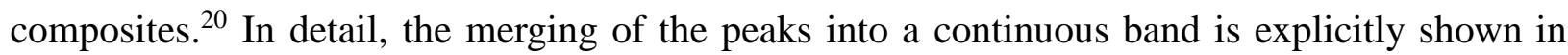
Fig. 3. where we increased the layer to $N=50$. The fcc lattice is taken as an example and we present both the longitudinal (left panel) and the transverse (right panel) results. The peaks in Fig.2(c) and Fig. 2(f) are distinctly smeared out in Fig. 3(a) and Fig. 3(b), respectively. The transverse results [see Fig. 3(c) and (d)] in the presence of the gradient is slightly different to that in the longitudinal case [Fig. 3 a) and (b)], but still retain the broadened bands. We ascribe this to the fact that the layer-to-layer interactions fall off exponentially due to the screening effect in the lattice, ${ }^{15}$ therefore give no much layer-structure-dependent difference in the two cases. Note that the longitudinal and transverse results of crystals in homogeneous host (dotted and dashed lines in Fig. 3) do not differ much as well.

In conclusion, we theoretically exploit the optical resonant enhancement due to lattice effect 
and gradient effect in colloidal crystals, which are made out of suspended metallic nanoparticles in a graded-index host. The gradient in the fluid and the colloid structure are easily subjected to tunability, for example, the structure transformation might be induced by electrorheological effects or by self-assembly of two kinds of particles with biochemically different surface properties, etc. In addition, one can also use metal-covered mangetic nanoparticles and control the suspension structure by external magnetic field, consequently realizing magneto-controlled optical properties. ${ }^{21}$ In this case, the electro-magnetorheological effects is a good candidate as well. Devices that could benefit from these materials include optical switches, optical limiters, as well as biosensors, etc.

This work was supported by the RGC Earmarked Grant. We thank Dr. J. P. Huang for critical reading of the manuscript.

1 Optical Properties of Nanostructured Random Media, edited by V. M. Shalaev (Springer, Berlin, 2002).

2 V. M. Shalaev, Phys. Rep. 272, 61 (1996); A. K. Sarychev and V. M. Shalaev, Phys. Rep. 335, 275 (2000), and references therein.

3 G. L. Fischer, R. W. Boyd, R. J. Gehr, S. A. Jenekhe, J. A. Osaheni, J. E. Sipe, and L. A. Weller-Brophy, Phys. Rev. Lett. 74, 1871 (1995); R. S. Bennink, Y. K. Yoon, R. W. Boyd, and J. E. Sipe, Opt. Lett. 24, 1416 (1999); N. N. Lepeshkin, A. Schweinsberg, G. Piredda, R. S. Bennink, and R. W. Boyd, Phys. Rev. Lett. 93, 123902 (2004).

4 L. Dong, G. Q. Gu, and K. W. Yu, Phys. Rev. B 67, 224205 (2003); L. Gao, J. P. Huang, and K. W. Yu, ibid. 69, 075105 (2004); J. P. Huang and K. W. Yu, Appl. Phys. Lett. 85, 94 (2004); J. P. Huang, L. Dong, and K. W. Yu, Europhys. Lett. 67, 854 (2004); J. P. Huang and K. W. Yu, Opt. Lett. 30, 275 (2005).

5 A. M. Freyria, E. Chignier, J. Guidollet, and P. Louisot, Biomaterials 12, 111 (1991).

6 H. Karacali, S. M. Risser, and K. F. Ferris, Phys. Rev. E 56, 4286 (1997).

7 U. Levy, M. Nezhad, H. C. Kim, C. H. Tsai, L. Pang, and Y. Fainman, J. Opt. Soc. Am. A 22, 724 (2005) and references therein.

8 Colloids and colloid assemblies, edited by F. Caruso (Wiley-VCH, Weinheim, 2004).

9 D. Y. Wang, J. Li, C. T. Chan, V. Salgueiriño-Maceira, L. M. Liz-Marzán, S. Romanov, and F. Caruso, Small 1, 122 (2005) 
10 K. P. Velikov, C. G. Christova, R. P. A. Dullens, and A. van Blaaderen, Science 296, 106 (2002)

11 T. Gong and D. W. M. Marr, Appl. Phys. Lett. 85, 3760 (2004)

12 S. O. Lumsdon, E. W. Kaler, J. P. Williams, and O. D. Velev, Appl. Phys. Lett. 82, 949 (2003)

13 See, for example, S. Riikonen, I. Romero, and F. J. García de Abajo, Phys. Rev. B 71, 235104 (2005)

14 C. K. Lo and K. W. Yu, Phys. Rev. E 64, 031501 (2001).

15 J. Lekner, Physica A 157, 826 (1989); ibid. 176, 485 (1991); S. V. Lishchuk, Mol. Phys. 100, 3789 (2002).

16 G. Q. Gu and K. W. Yu, Phys. Rev. B 46, 4502 (1992)

17 X. C. Zeng, D. J. Bergman, P. M. Hui, and D. Stroud, Phys. Rev. B 38, 10970 (1988)

18 A. Sharkawy, D. Pustai, S. Y. Shi, D. W. Prather, S. McBride, and P. Zanzucchi, Opt. Express 13, 2814 (2005).

19 J. J. Xiao, J. P. Huang, and K. W. Yu, Phys. Rev. B 71, 045404 (2005).

20 L. Dong, M. Karttunen, and K. W. Yu, Phys. Rev. E 72, 016613 (2005)

21 J. P. Huang and K. W. Yu, Appl. Phys. Lett. 86, 041905 (2005) 


\section{Figure Captions}

FIG. 1: Schematic diagram of the colloidal crystal immersed in a graded host medium. In the numerical calculations $a=b$ is assumed, which forms a square dipole lattice in $x y$-plane, while $c$ is the lattice constant in the direction of $z$-axis.

FIG. 2: The effective linear response $\operatorname{Im}\left(\epsilon_{\text {eff }}\right)$ and third-order nonlinearity enhancement factor $|\gamma|$ of the periodic colloidal nanoparticles immersed in a graded host fluid (solid line), and in homogeneous host fluids with dielectric constant $\epsilon_{2}=2.25$ (dashed line) and $\epsilon_{2}=1.0$ (dotted line), respectively; (a), (d) for bct, (b), (e) for bcc; and (c), (f) for fcc, respectively. Parameters: $\Gamma=0.02 \omega_{p}, N=25, a=1$, and $\left|\mathbf{E}_{0}\right|=1$.

FIG. 3: Same as Fig. 2 but with totally $N=50$ layers for fcc lattice. (a) and (b) the longitudinal case; (c) and (d) the transverse case. 


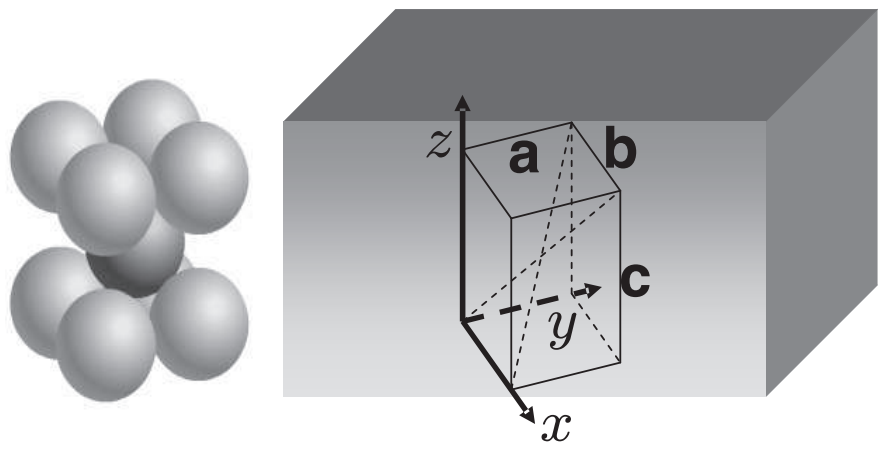

Fig.1./Xiao and $\mathrm{Yu}$ 

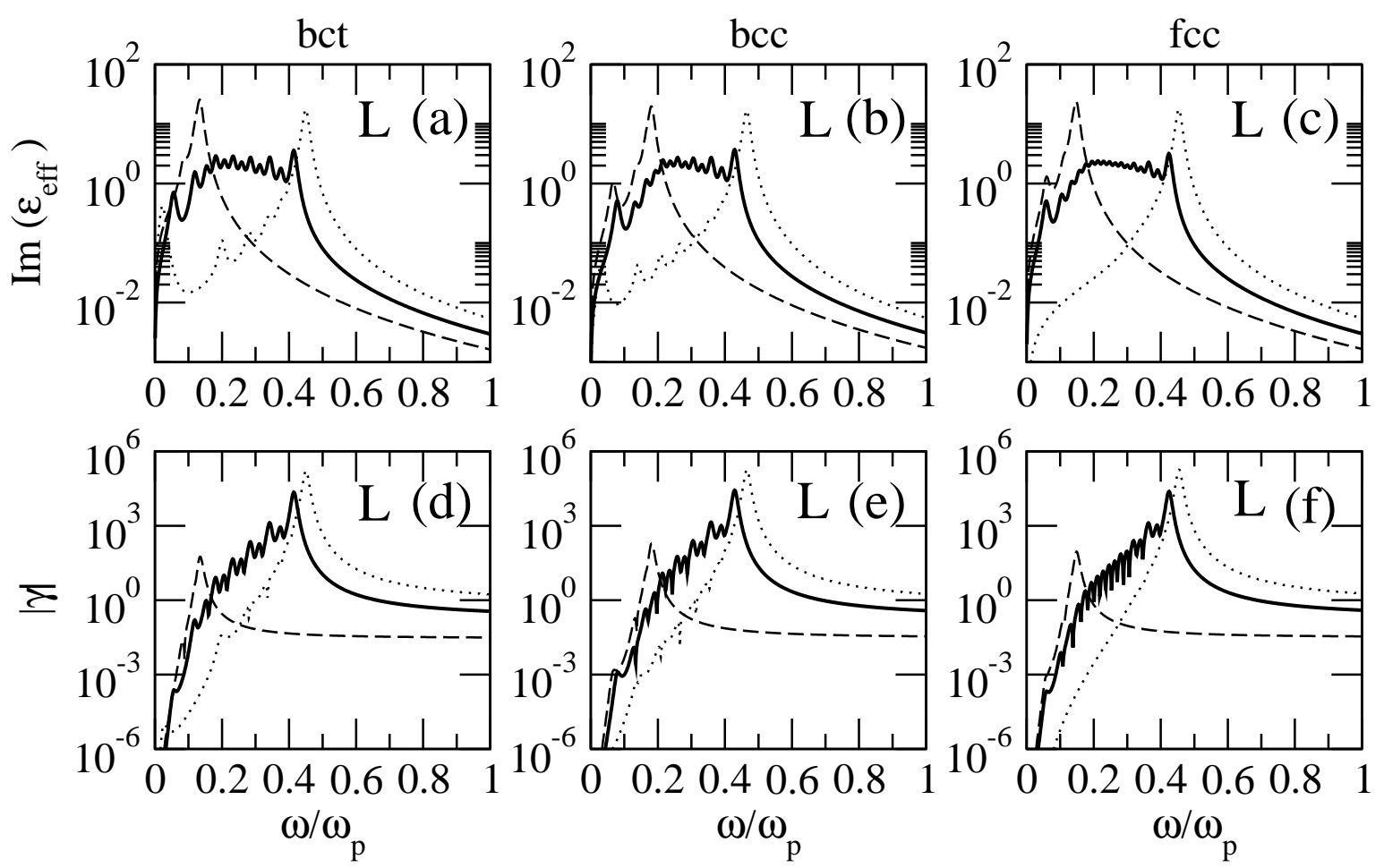

Fig.2./Xiao and $\mathrm{Yu}$ 

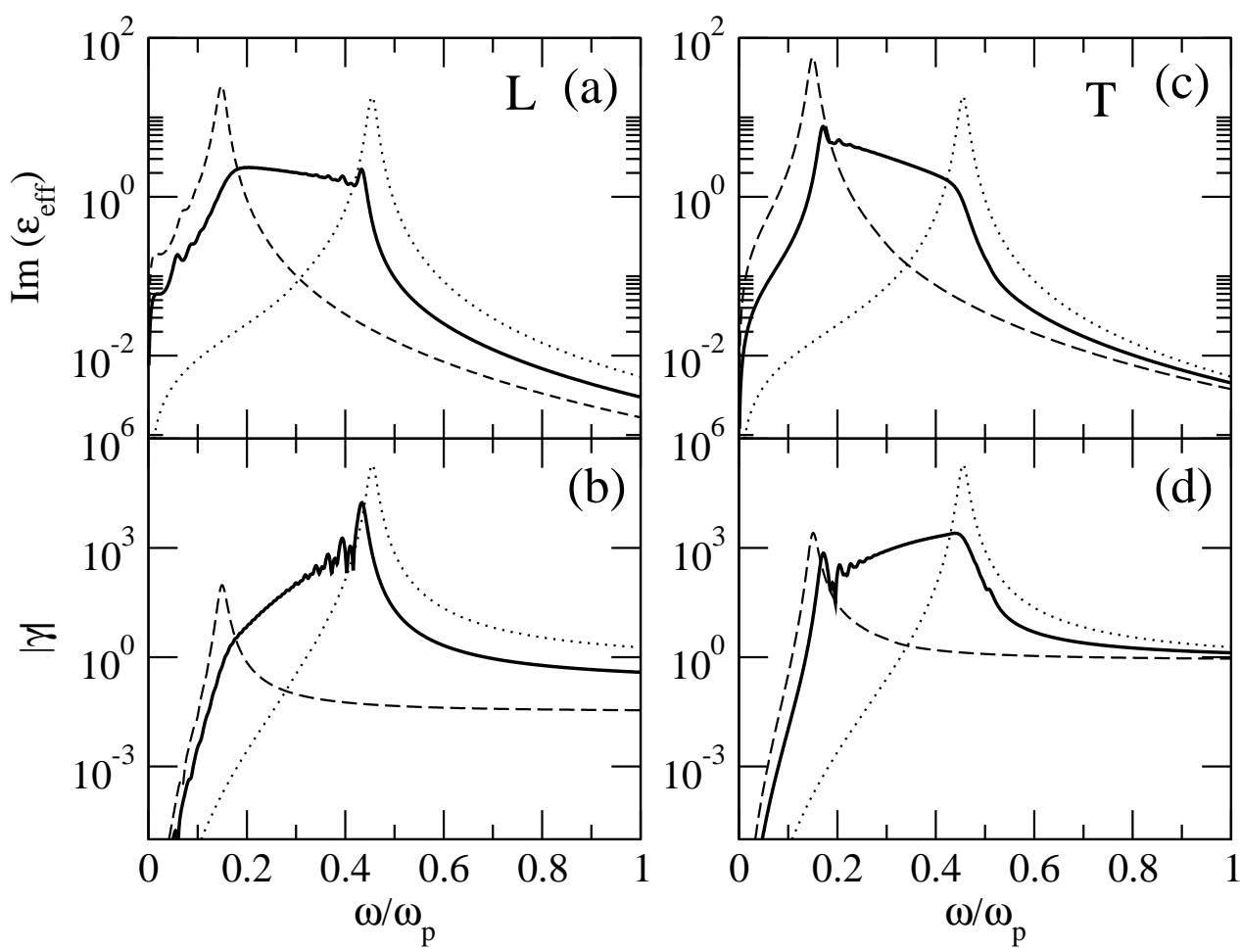

Fig.3./Xiao and $\mathrm{Yu}$ 